\title{
Archipel
}

ARCHIPEL Études interdisciplinaires sur le monde insulindien

$88 \mid 2014$

Varia

\section{Songs of Memory in Islands of Southeast Asia}

\section{Hélène Bouvier}

\section{OpenEdition}

Journals

Édition électronique

URL : http://journals.openedition.org/archipel/548

DOI : 10.4000/archipel.548

ISSN : 2104-3655

\section{Éditeur}

Association Archipel

\section{Édition imprimée}

Date de publication : 10 octobre 2014

Pagination : 228-230

ISBN : 978-2-910513-71-9

ISSN : 0044-8613

Référence électronique

Hélène Bouvier, "Songs of Memory in Islands of Southeast Asia », Archipel [En ligne], 88 | 2014, mis en ligne le 10 septembre 2017, consulté le 25 septembre 2020. URL : http://journals.openedition.org/ archipel/548; DOI : https://doi.org/10.4000/archipel.548 
alternés. Asie du Sud-Est. Vingt-trois ans après, tout cela est devenu possible.

Un ouvrage associant les supports audio-visuels et les possibilités de répétition qu'ils offrent, associés aux analyses des actions en une suite d'instants vécus par les hommes et les femmes, leurs actes, captés par le regard de l'ethnographe et la mémoire du film n'en serait que plus convaincant pour le lecteur. De plus, il permettrait de conserver un patrimoine vocal, gestuel, kinésique, chorégraphique et scénographique. Il s'agit bien ici de pratiques performatives dans des contextes rituels ou des expressions théâtrales spécifiques. Or le mot performance, pas plus que les références fondamentales qu'il implique, notamment aux ÉtatsUnis, mais aussi en Europe (Ethnoscénologie, 1995, J.-M. Pradier et les recherches à Paris 8, dans le sillage de J. Grotowski, le Théâtre des sources) n'apparaissent pas dans ce livre, alors qu'H. Bouvier employait ce terme dans les années 1990. Les travaux des anthropologues en France sur les performances rituelles et/ou théâtrales ont considérablement progressé depuis lors. L'omission de ce terme est-elle délibérée?

Nicole Revel

Nicole Revel (ed.), Songs of Memory in Islands of Southeast Asia, Cambridge Scholars Publishing, Newcastle upon Tyne, 2013, xvi+320 pages, ill. ISBN 978-1-4438-4445-1.

Cet ouvrage collectif fait suite à la conférence « Songs of Memory : Safeguarding Cultural Heritage at the Turn of the $21^{\text {st }}$ century », qui s'est tenue du 20 au 22 janvier 2011 à Ateneo de Manila University, aux Philippines, à l'occasion de l'inauguration du site libre d'accès sur la toile : Philippines Epics and Ballads Archives. Il est un témoin de plus de l'engagement de Nicole Revel dans le domaine de ce qu'elle appelle les «Littératures de la voix », mais également dans celui, plus récent, des « Digital Humanities » qui paraissent pour l'heure pouvoir garantir la sauvegarde de pans entiers de cette mémoire aurale et orale de l'humanité. En construisant le site, à Ateneo de Manila University, c'est tout un patrimoine intangible qui, au-delà des seules archives, se constitue et reflète le souci indéfectible de Nicole Revel pour une transmission à la fois académique, culturelle et éducative. Au-delà des Philippines, le présent ouvrage réunit les contributions d'une douzaine de chercheurs qui se consacrent au domaine des expressions musicales, narratives et rituelles, à travers l'aire de l'Asie du Sud-Est insulaire, allant du travail de collecte de terrain, à celui de la transcription et de la traduction, jusqu'à la recherche de supports multimédias modernes et de versions digitalisées.

Le livre est organisé en trois parties : " Chanted Narratives Intangible Heritage in Today's World », «The Voice, The Music, The Word », et «Interpreting as an Art ». La première partie réunit trois contributions à teneur théorique, qui cherchent à établir le lien entre les données recueillies sur le terrain et leur intégration dans le domaine des recherches sur l'oralité. La deuxième partie compte quatre contributions consacrées à l'étude des relations entre les mots, la voix et le chant, travaux monographiques d'anthropologie et d'ethnomusicologie, attentifs aux langues vernaculaires et aux catégories émiques. La troisième partie rassemble cinq articles dévolus à l'interprétation des épopées à travers toute cette aire culturelle de l'Asie du Sud-Est insulaire, et sur de longues périodes.

C'est l'anthropologue Fernando Zialcita qui, avec " Chanted Landscapes », ouvre le recueil en posant la question de la relation entre tangible et intangible, à travers l'étude 
d'extraits d'épopées qui, en faisant apparaître dans leur narration des éléments matériels et tangibles mais disparus ou oubliés, et en les associant parfois à des sons, leur redonne vie pour l'auditeur, assurant ainsi leur conservation grâce à l'intangible et immatériel. Pour ses réflexions sur l'oralité ou la voix, l'auteur convoque les travaux de Merleau-Ponty, Hegel, Bachelard et Meschonnic. Ensuite, Leovino Garcia, dans « Paul Ricoeur's Hermeneutics of Capable Human Being : Ethical Implications of Epic Narratives », applique la théorie narrative de la Mimesis du philosophe français Paul Ricoeur au champ de la tradition orale, à partir d'exemples tirés d'épopées, pour en démontrer la dimension éthique. Dans sa contribution intitulée " Vivid and Virtual Memory », Nicole Revel, linguiste et anthropologue, éditrice de ce volume, compare deux compositions poétiques des Philippines et s'interroge sur les relations entre mémoire et créativité dans les cultures de tradition orale, et l'arrivée de la culture multimédia. Des appendices retraçant l'historique des études sur les épopées aux Philippines et l'élaboration des archives multimédia, ainsi que des cartes et des schémas d'analyse des structures d'épopées, complètent le panorama de cette vaste entreprise menée par l'auteur depuis plusieurs décennies.

La deuxième partie commence par trois chapitres écrits par des ethnomusicologues, qui reflètent le souci partagé de prendre en compte à la fois les paroles et la musique, la matière vocale et les mots. Dans "Mangalimog Ako: Finding One's Voice in Sugidanon (Epic Chanting) ", Maria Christine Muyco, explique les principes et la mise en pratique du limog et du sibod, utilisés dans la création vocale des Panay Bukidnon du centre des Philippines, ainsi que l'interdépendance de la voix et des mots. Dans « Speech and Songs in the Toraja Highlands ", Dana Rappoport étudie comment la musique s'enracine dans le discours rituel chez les Toraja du sud de Sulawesi en Indonésie, à partir des rituels du côté du Soleil Levant et du Soleil Couchant, et d'un poème funéraire hagiographique, tout en exposant la construction et l'utilisation d'archives multimédia qui y sont dédiées, formant une «mémoire musicale digitalisée ». Enfin Manolete Mora s'intéresse, dans « Madness and Recovery : Rite, Renewal, and the Ring Form in T'boli Epic Song », à l'organisation temporelle de la narration et de la musique d'un épisode de l'épopée Tudbulul des T'boli du sud-ouest de Mindanao aux Philippines.

La contribution de l'anthropologue Rosario Bona de Santos del Rosario, dans "The Chanted Ifugao Alim and Hudhud : Ritual-Drama and Heroic Stories », dans la suite du précédent chapitre, compare les chants cousins Alim et Hudhud des Ifugao du nord de Luzon, aux Philippines, tous deux dévolus au thème des aléas de la relation amoureuse ou maritale, et chantés en alternant soliste et choeur.

La troisième partie s'ouvre sur une autre étude de ce genre Hudhud et s'enrichit d'une nouvelle dimension. Dans «Epic as a Means to Control the Memory and Emotions of Gods and Humans : Ritual Implications of the Hudhud Epics among the Ifugao and the Kalanguya », l'anthropologue Maria Stanyukovich montre comment cette épopée met en jeu le lien de cette société avec son panthéon et organise la réconciliation nécessaire entre les parents d'un défunt et l'esprit du mort, et vise le juste équilibre des émotions.

Chez les Moken, nomades marins de l'archipel des Mergui, l'anthropologue Jacques Ivanoff, dans « From the Founding Epic to a Millennarian Unknown, Moken' Answer to Contemporary History ", s'intéresse à l'émergence et à l'adaptation d'une nouvelle épopée mettant en scène des missionnaires, ainsi qu'au syncrétisme à l'œuvre sans relâche entre mythes et rituels, dans un environnement social, religieux et écologique, qui ne cesse d'être bouleversé. Le linguiste Aone van Engelenhoven, dans « The Spoor of the Mythical Sailfish : 
Narrative Topology and Narrative Artefacts in Southwest Maluku (Indonesia) and Tutuala (East-Timor) ", compare les versions de ce mythe entre les îles du sud des Moluques en Indonésie, et la région de Tutuala à Timor-Est, connues inégalement, et selon des impératifs rituels différents, mais démontrant des traditions de conte similaires et faisant référence à un réseau économique ancien commun.

Une approche plus historique conduit le chapitre suivant, «Epics in the Early Spanish Philippines Revisited », dans lequel Brandon Reilly dépouille les descriptions laissées par les premiers Espagnols qui ont assisté, à la fin du XVI ${ }^{\mathrm{e}}$ et au début du XVII ${ }^{\mathrm{e}}$ siècles à des séances d'épopées chantées aux Philippines, et en relatent les évaluations contrastées, souvent déjà curieuses d'histoire et de culture locales. Le volume se clôt sur la contribution de Muhammad Haji Salleh, «Hang Tuah, the Culture Hero : Interpreting Memory », qui fait part de l'émotion toujours vivace qu'inspire aux Malais leur héros d'épopée, sans cesse réinterprété à la lumière des changements de leur société.

Ce volume fait la démonstration éclatante de la richesse et de la complexité des recherches menées dans le domaine de l'oralité, tout particulièrement dans cette région de l'Asie du SudEst, et encouragera les spécialistes de toutes les disciplines des sciences humaines, ou de telle ou telle société de cette aire, non seulement à en lire les chapitres, mais encore à consulter les sources et références qui y sont abondamment citées. Pour reprendre les mots optimistes de Nicole Revel (p. xvi) : " Même si les forces de l'oubli et de la destruction sont nombreuses dans le monde d'aujourd'hui, les forces de la mémoire et de la créativité les contrebalancent d'une manière authentique, contemporaine et pourtant traditionnelle », et l'auteur d'appeler, au-delà de l'enseignement des langues en danger de disparition, à offrir l'espace virtuel cybernétique à toutes leurs expressions, y compris artistiques.

On peut se reporter au site sur la toile : http://epics.ateneo.edu/epics

Hélène Bouvier

Ardian Purwoseputro, Wayang Potehi of Java 布袋戏, Jakarta, Afterhours Books, 2014, 290 p., 2 maps, sumptuous illustrations. Foreword by Prof. Leo Suryadinata. ISBN : 978602-97507-9-9

Wayang Potehi, is a popular form of puppet theatre which comes from Southern Fujian province. The term potehi (in Mandarin: budai xi) literally means "glove puppet theatre". No information is available about its historical development in Fujian. Xie Zhaozhi 谢肇淛 (1567-1624) in his Wu zazu 五杂沮 (“Five assorted offerings”, first ed. 1616, ed. Minguo 24, I. p. 213) alludes to the taste of Southern Chinese for "puppetry" (here called kuilei 傀儡, presumably a loanword, which can be traced back at least to the Tang Dynasty), says that it is of foreign origin (huxi 胡戏). Unfortunately, he does not provide further comments on the various forms of puppet theatre then in vogue. As for Indonesia, the early European travellers who visited the island only recorded plays performed by human actors. So far, the oldest allusion to Potehi performances in Java seems to emanate from Chinese records dating back to the last decades of the $18^{\text {th }}$ century.

The book by Ardian Purwoseputro is aimed at providing an overview of the ups and downs of wayang Potehi in Java up to the present. After a brief introductory chapter on puppetry in Fujian, as perceived through various legends, the author focuses on its second 